\title{
Medición volumétrica de grasa visceral abdominal con resonancia magnética y su relación con antropometría, en una población diabética
}

\author{
CRISTÓBAL SERRANO GARCÍA ${ }^{1,5}$, FRANCISCO BARRERA ${ }^{2}$, \\ PILAR LABBÉ ${ }^{2}$, JESSICA LIBERONA ${ }^{3}$, MARCO ARRESE2, \\ PABLO IRARRÁZAVAL ${ }^{1,4}$, CRISTIÁN TEJOS ${ }^{1,4}$, SERGIO URIBE ${ }^{1,5}$
}

\section{Quantification of visceral adipose tissue using magnetic resonance imaging compared with anthropometry, in type 2 diabetic patients}

Background: Visceral fat accumulation is associated with the development of metabolic diseases. Anthropometry is one of the methods used to quantify it. aim: to evaluate the relationship between visceral adipose tissue volume (VAT), measured with magnetic resonance imaging (MRI), and anthropometric indexes, such as body mass index (BMI) and waist circumference (WC), in type 2 diabetic patients (DM2). Patients and Methods: Twenty four type 2 diabetic patients aged 55 to 78 years (15 females) and weighting 61.5 to $97 \mathrm{~kg}$, were included. The patients underwent MRI examination on a Philips Intera ${ }^{\circledR}$ 1.5T MR scanner. The MRI protocol included a spectral excitation sequence centered at the fat peak. The field of view included from L4-L5 to the diaphragmatic border. VAT was measured using the software Image $J^{\circledast}$. Weight, height, BMI, WC and body fat percentage (BF\%), derived from the measurement of four skinfolds with the equation of Durnin and Womersley, were also measured. The association between MRI VAT measurement and anthropometry was evaluated using the Pearson's correlation coefficient. Results: Mean VAT was $2478 \pm$ $758 \mathrm{ml}$, mean BMI $29.5 \pm 4.7 \mathrm{~kg} / \mathrm{m}^{2}$, and mean WC was $100 \pm 9.7 \mathrm{~cm}$. There was a poor correlation between VAT, BMI $(r=0.18)$ and WC $(r=0.56)$. Conclusions: BMI and WC are inaccurate predictors of VAT volume in type 2 diabetic patients.

(Rev Med Chile 2012; 140: 1535-1543).

Key words: Anthropometry; Diabetes Mellitus, Type 2; Intra-abdominal fat; Magnetic Resonance Imaging.

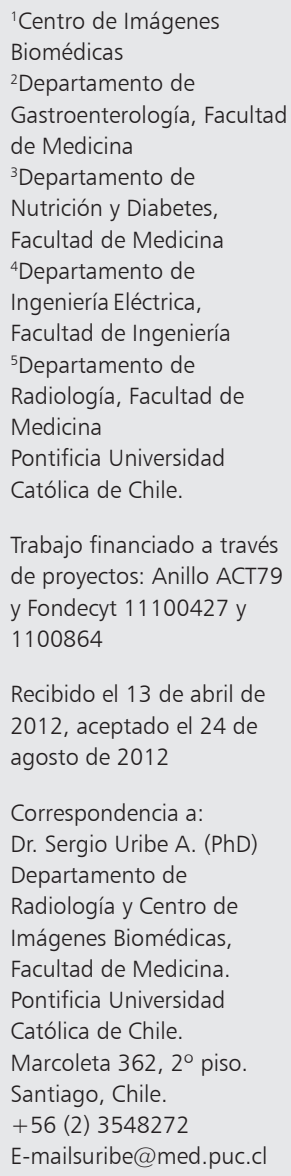

L a obesidad es un factor de riesgo importante en numerosas patologías, tales como resistencia insulínica, síndrome metabólico, diabetes mellitus tipo 2 (DM2), hipertensión arterial, dislipidemia, enfermedad cardiovascular y enfermedad hepática por hígado graso, entre otras $^{1-5}$.

Para evaluar los riesgos de la obesidad, se hace énfasis en la distribución que presenta la grasa abdominal, la que se puede clasificar en tejido adi- poso subcutáneo (TAS) o tejido adiposo visceral $(\mathrm{TAV})$. Esta última es la de mayor relevancia ${ }^{6-9}$, ya que es más activa metabólicamente y tiene mayor incidencia en el aumento de la producción de adipoquinas, la actividad pro-inflamatoria y la alteración de la sensibilidad a la insulina ${ }^{10,11}$.

Tradicionalmente, la antropometría se ha usado como método clínico para la evaluación de la obesidad $^{12,13}$. El parámetro más usado ha sido la circunferencia abdominal (CA), la cual es parte de 
los criterios diagnósticos del síndrome metabóli$\mathrm{CO}^{14}$. Sin embargo, hay cierta evidencia que la CA por sí sola no es un buen predictor de la cantidad de $\mathrm{TAV}^{15,16}$, y que las mediciones son imprecisas ${ }^{17,18}$.

Desde hace algunos años se han utilizado métodos más precisos para cuantificar la grasa intra abdominal basados en imágenes. Dentro de estos, están la tomografía axial computarizada (TAC) y la resonancia magnética $(\mathrm{RM})^{19,20}$, siendo esta última la que ha ganado más aceptación, dado que no utiliza radiación ionizante ${ }^{21}$.

A pesar de que el "estándar de oro" para cuantificar grasa intra abdominal es la espectroscopia por RM, la cuantificación de TAV y TAS se realiza habitualmente con secuencias de RM potenciadas en $\mathrm{T}^{22}$. Esto se debe a que la espectroscopia sólo permite mediciones locales (pocos voxeles). La cuantificación con alta resolución espacial de grandes volúmenes no es factible debido a la lentitud de la técnica de espectroscopia ${ }^{23}$. Por otra parte, las imágenes de RM potenciadas en T1 pueden producir sobreestimaciones en la cuantificación de los volúmenes de grasa ${ }^{24}$.

Se han desarrollado técnicas alternativas de RM para cuantificar volúmenes de grasa de manera más precisa. Entre ellas están: secuencias por excitación espectral de grasa, el método Dixon ${ }^{25,26}$, y el método IDEAL ${ }^{\circledR}$ (Iterative Decomposition with Echo Asymetry and Least squares) $)^{27,28}$.

Generalmente, la medición de grasa a partir de imágenes de RM se realiza en forma manual, proceso que es engorroso, demandante de tiempo, y puede tener alta variabilidad inter e intra observador. Por ello, han surgido nuevas aplicaciones computacionales, que permiten hacer este proceso de manera más confiable y en menor tiempo ${ }^{29-32}$.

Las mediciones volumétricas de grasa intra abdominal en pacientes obesos (utilizando RM) tienen una baja correlación con las medidas antropométricas ${ }^{33}$. De igual manera, se ha descartado la relación entre parámetros biométricos y el porcentaje de grasa en el hígado. Esto ha sido verificado mediante biopsia hepática en pacientes con enfermedades hepáticas como hígado graso y fibrosis hepática ${ }^{34}$. Sin embargo, esta relación ha sido poco estudiada en pacientes con DM2, quienes pueden tener fenotipo delgado ${ }^{35-38}$.

En pacientes con DM2 se ha comunicado que la presencia de hígado graso, como condición inicial, podría conllevar a esteatohepatitis y cirrosis ${ }^{39-40}$. Además, estudios en población general, muestran que la presencia de TAV podría tener un rol en la patogénesis de HGNA (hígado graso no alcohólico), así como en el desarrollo de esteatohepatitis y fibrosis hepática ${ }^{41-42}$. Luego, es importante cuantificar correctamente la cantidad de TAV, y utilizar estas mediciones como un eventual predictor de riesgo que permita pesquisar y tratar oportunamente las complicaciones hepáticas en el subgrupo de sujetos de mayor riesgo.

El objetivo del presente trabajo es evaluar, en pacientes con DM2, la relación que pudiera existir entre la cantidad de TAV y los parámetros antropométricos que se utilizan en forma estándar para predecir volumen de grasa abdominal. La comparación la realizamos utilizando imágenes de RM con excitación espectral de grasa. Para mejorar la precisión y reducir los tiempos de proceso, utilizamos un software semi-automático de procesamiento de imágenes para cuantificar los volúmenes de TAV.

\section{Material y Método}

\section{Sujetos}

Se incluyeron en el protocolo 24 pacientes diabéticos tipo 2, mayores de 55 años en control médico regular. El diagnóstico de DM2 se basó en los criterios $\mathrm{ADA}^{43}$. Esto es cumplir al menos uno de los siguientes indicadores: (i) $\mathrm{HbA} 1 \mathrm{C} \geq 6,5 \%$; (ii) glicemia de ayuno $\geq 126 \mathrm{mg} / \mathrm{dL}$; (iii) test de tolerancia a $75 \mathrm{~g}$ de glucosa con glicemia $\geq 200$ $\mathrm{mg} / \mathrm{dL}$ a las $2 \mathrm{~h}$ post carga; (iv) glicemia $>200 \mathrm{mg} /$ dL con síntomas de hiperglicemia, (i.e. polidipsia, polifagia, poliurea, pérdida de peso).

Se consideraron los siguientes criterios de exclusión: Tener enfermedad hepática conocida, excepto HGNA, pruebas hepáticas alteradas (transaminasas mayores que 3 veces valor normal (> $159 \mathrm{UI} / \mathrm{L})$, FA y GGT mayores que 2 veces valor normal (> 199 UI/L y > 99 UI/L respectivamente), consumo de alcohol mayor a $30 \mathrm{~g} /$ día en hombres y $20 \mathrm{~g} /$ día en mujeres) y contraindicación de realizarse una RM. El protocolo fue aprobado por el comité de ética y se obtuvo consentimiento informado de todos los pacientes.

\section{Adquisición de imágenes}

Los pacientes se sometieron a un examen de $\mathrm{RM}$ en un resonador Philips Intera ${ }^{\circledR}$ de 1.5T. El protocolo incluyó distintas secuencias diagnósticas y además se agregó una secuencia espectral con 
excitación selectiva de grasa con los siguientes parámetros; TR $1.400 \mathrm{~ms}$, TE $101 \mathrm{~ms}$, ángulo de excitación $90^{\circ}$, NSA 1 , espesor de corte $7 \mathrm{~mm}$, campo de visión de 480 x 384 mm y matriz de $256 \times 256$ pixeles.

Un total de 32 cortes transversales, sin espacios entre cortes, fueron adquiridos en cada paciente, utilizando las vértebras L4-L5 como referencia anatómica y punto de partida, cubriendo en promedio en dirección craneal hasta la cúpula del diafragma. Para reducir artefactos de movimiento, todo el volumen fue adquirido en 3 apneas que duraron aproximadamente $18 \mathrm{seg}$ cada una.

\section{Segmentación}

Las imágenes adquiridas fueron analizadas usando el software Image ${ }^{\circledR}$ (National Institute of Health, Bethesda, MD) (http://rsbweb.nih.gov/ij/) por un observador, el cual ejecutó los siguientes pasos para segmentar TAV y TAS:

1. Transformar cada imagen de RM (Figura 1a) a una imagen binaria (blanco o negro, Figura 1b), siendo blanco los pixeles de grasa y negro los que no contienen grasa. La binarización se realizó comparando los niveles de gris de la imagen original contra un valor umbral escogido heurísticamente para cada paciente.

2. Los artefactos presentes en las imágenes, producto de inhomogeneidades de campo o susceptibilidades magnéticas fueron corregidos manualmente, al igual que la exclusión de estructuras óseas. Esto se realizó comparando las imágenes originales con los cortes superiores e inferiores de cada imagen. Un ejemplo de un área afectada por artefactos y el resultado de la corrección se indican con flechas en las Figuras 1a y $1 \mathrm{~b}$.

3. Se cuantificó el volumen total de grasa abdominal (TAV+TAS) como el número de pixeles blancos de todas las imágenes binarias, multiplicando por el volumen de cada pixel (i.e. el espesor de corte por el área de un pixel).

4. Para separar TAV y TAS se delineó (en forma semi-automática y en cada una de las imágenes binarias) el contorno interno de la cavidad visceral. De esta forma se generó un área delimitada para medir el volumen de TAV (Figura 1c).

5. Se cuantificó el volumen total de TAV de igual forma que en el paso 3, pero considerando sólo el área interna de los contornos definidos en el paso 4 .

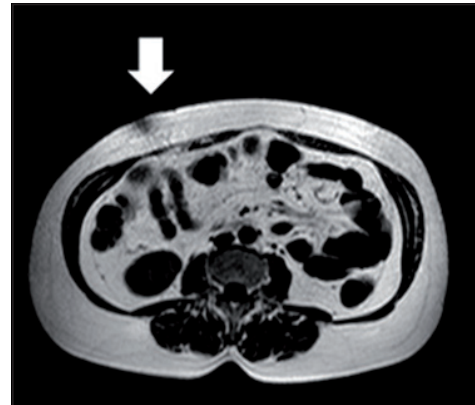

a
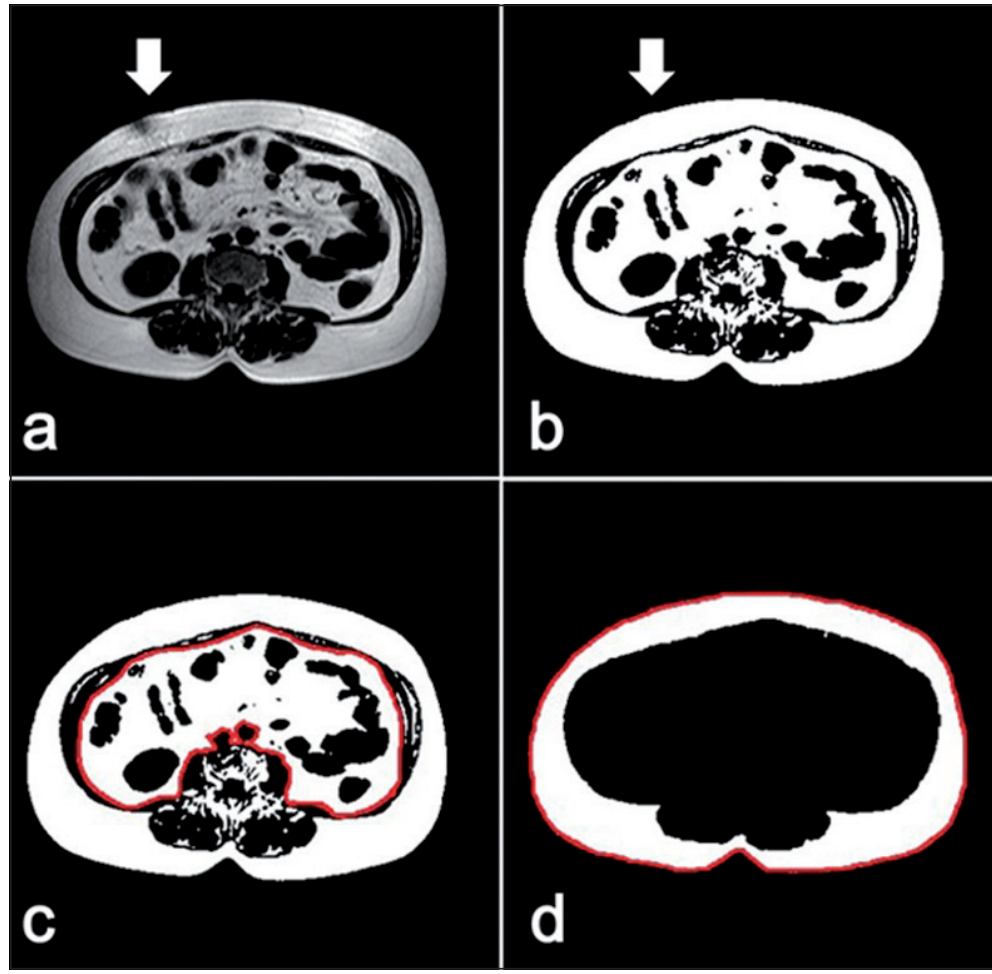

Figura 1. Método de determinación de volumen adiposo visceral y subcutáneo. (a) Imagen de RM original. (b) Mediante procesamiento de la imagen con el software Image J se obtiene una imagen binaria que destaca el tejido adiposo en blanco. (c) En forma manual se corrigen artefactos, se excluyen tejido óseo y se mide la superficie de tejido adiposo visceral. (d) Mediante la sustracción de la superficie de grasa visceral a la superficie grasa total se obtiene la superficie de tejido adiposo subcutánea. Las flechas en (a) y (b) indican un artefacto en la imagen y su corrección, respectivamente. A partir del volumen de cada pixel (determinado por el número de cortes de RM) se calcula el volumen de grasa visceral (TAV) o subcutánea (TAS). 
6. Para medir TAS, se restó el volumen de TAV al total de la grasa abdominal (TAV+TAS). Esto equivale a hacer los cálculos de volumen excluyendo la región de TAV en cada corte (Figura 1d).

El proceso de análisis de las imágenes duró aproximadamente 50 min por paciente.

\section{Antropometría}

Cada paciente se sometió a un estudio antropométrico, obteniéndose datos de peso, talla, índice masa corporal (IMC), porcentaje masa grasa por impedanciometría (MGI\%), circunferencia abdominal (CA), pliegue tricipital (PT), pliegue bicipital (PB), pliegue supra ilíaco (Psp) y pliegue subescapular (Psb).

A partir de los 4 pliegues medidos se calculó la densidad corporal específica (D) mediante la ecuación propuesta por Durnin y Womersley ${ }^{44}$ : D $=\mathrm{C}-\left(\mathrm{M} \times \log 10 \sum\right.$ cuatro pliegues $)$, utilizando los coeficientes $\mathrm{C}$ y $\mathrm{M}$ de la suma de los cuatro pliegues reflejados en las tablas desarrolladas por estos autores para cada sexo y en forma específica para edad mayor a 50 años. Con ello se calculó el porcentaje de masa grasa por pliegues (MGP\%) mediante la ecuación de Siri ${ }^{45}: \%$ grasa corporal $=[(4,95 /$ densidad $)-4,5] \times 100$.

\section{Método estadístico}

Todos los análisis fueron realizados usando el software SPSS 19.0 (SPSS Inc., Chicago, IL). Se determinó la distribución normal de los datos mediante las pruebas estadísticas de KolmogorovSmirnov y Shapiro-Wilk. Promedios y desviaciones estándar fueron usados para describir la muestra en el estudio. En cada paciente, se calculó la correlación de Pearson (r), entre TAS, TAV, y TAV+TAS asociado a cada variable antropométrica.

Se estableció $\mathrm{P}<0,05$ para indicar significancia estadística de la hipótesis evaluada: la existencia de alguna asociación entre el volumen de grasa (medido en las imágenes de RM) y los parámetros antropométricos evaluados.

\section{Resultados}

En la Tabla 1 se muestra un resumen descriptivo de todas las variables antropométricas y de grasa medidas por RM. Un total de 24 pacientes ( 9 hombres, 15 mujeres, edad promedio: 62 años, rango: $55-78$ años, peso promedio: $77,2 \mathrm{~kg}$, rango: 61,5-97 kg), se sometieron a una RM de abdomen.

De los 24 pacientes, $37,5 \%$ fue catalogado como obeso (IMC $\geq 30$ ). Del total de mujeres (promedio IMC: $31 \pm 4,8$, rango: $23,1-37,6$ ), 53,3\% era obesa, y del total de hombres (promedio IMC: $27,02 \pm 3,69$, rango: $22,6-35,1$ ) sólo $11,1 \%$ era obeso. De acuerdo a la definición de síndrome metabólico ${ }^{14}, 33,3 \%$ de los hombres tenía un CA elevado $(>102 \mathrm{~cm})$ y $80 \%$ de las mujeres tenía un CA elevado $(>88 \mathrm{~cm})$. En promedio, incluyendo hombres y mujeres, el CA fue de 100,28 \pm 9,74 (rango $85-120 \mathrm{~cm}$ ).

Los volúmenes promedio (medidos a partir de las imágenes de RM) fueron: TAV $2.478 \pm 758$ cc (rango: $1.173-3.836 \mathrm{cc}$ ), TAS $3.236 \pm 1.481 \mathrm{cc}$ (rango: $1.162-7.548 \mathrm{cc}$ ) y TAV+TAS $5.715 \pm 1.660$ cc (rango: 3.413-10.504 cc).

Los resultados de la correlación de Pearson entre TAV, TAS y TAV+TAS frente a cada variable antropométrica se muestran en la Tabla 2.

Observamos una correlación significativa de moderada cuantía entre TAV y las variables antropométricas CA y peso, en una correlación $(r=0,56$ y $\mathrm{r}=0,42$ respectivamente, $\mathrm{p}<0,05)$.

Para TAS se encontró una mayor correlación con la mayoría de las variables antropométricas medidas $(\mathrm{p}<0,05)$. Sin embargo, en ninguna de

\section{Tabla 1. Estadística descriptiva. Descripción de cada variable en estudio. $n=24$}

\begin{tabular}{|lcc|}
\hline Variables & Media & $\begin{array}{c}\text { Desviación } \\
\text { estándar }\end{array}$ \\
\hline TAV (cc) & 2.478 & 758 \\
\hline TAS (cc) & 3.236 & 1.481 \\
\hline TAV+TAS (cc) & 5.715 & 1.660 \\
\hline Edad (años) & 61,79 & 6,01 \\
\hline Peso (kg) & 77,2 & 11,24 \\
\hline Talla (m) & 1,62 & 0,08 \\
IMC (kg/m²) & 29,5 & 4,78 \\
CA (cm) & 100,28 & 9,74 \\
\hline MGI\% (\%) & 39,49 & 9,28 \\
\hline MGP\% (\%) & 37,98 & 7,39 \\
\hline
\end{tabular}

TAV: tejido adiposo visceral. TAS: tejido adiposo subcutáneo. IMC: índice masa corporal. CA: circunferencia abdominal. (MGI\%) porcentaje masa grasa medida por impedanciometría. (MGP\%) porcentaje de masa grasa medida por pliegues cutáneos. 
ellas $r$ fue superior a 0,8 . Las correlación más alta fueron para IMC y MG\% con valores de $r$ iguales a 0,8 y 0,77 respectivamente, $\mathrm{p}<0,01$.

En cuanto a la suma de TAV+TAS, las correlaciones fueron similares a las obtenidas para TAS (ninguna de ellas con $r>0,8$ ).

Tabla 2. Análisis estadístico. Correlación de Pearson ( $r$ ) y valor-p, entre TAV, TAS, y TAV+TAS, para cada variable antropométrica

\begin{tabular}{|llll|}
\hline Variables & TAV & TAS & TAV+TAS \\
Peso & $r=0,42$ & $r=0,5$ & $r=0,66$ \\
& $p=0,03$ & $p=0,008$ & $p<0,001$ \\
IMC & $r=0,18$ & $r=0,8$ & $r=0,79$ \\
& $p=0,34$ & $p<0,001$ & $p<0,001$ \\
CA & $r=0,56$ & $r=0,6$ & $r=0,8$ \\
& $p=0,004$ & $p=0,002$ & $p<0,001$ \\
MGI\% & $r=-0,26$ & $r=0,77$ & $r=0,57$ \\
& $p=0,2$ & $p<0,001$ & $p=0,003$ \\
MGP\% & $r=-0,30$ & $r=0,71$ & $r=0,49$ \\
& $p=0,15$ & $p=0,001$ & $p=0,01$ \\
\hline
\end{tabular}

TAV: tejido adiposo visceral. TAS: tejido adiposo subcutáneo. IMC: índice masa corporal. CA:circunferencia abdominal. (MGI\%) porcentaje masa grasa medida por impedanciometría. (MGP\%) porcentaje de masa grasa medida por pliegues cutáneos.
Dada la relevancia de CA, medición que se incluye dentro de los criterios diagnósticos ATPIII de síndrome metabólico, se realizó una evaluación más detallada con respecto a esta variable (Figura 2).

Al comparar TAV con los subgrupos de pacientes con CA alto (definido como mayor a 102 $\mathrm{cm}$ en hombres y $88 \mathrm{~cm}$ en mujeres) o bajo (Tabla 3 ), se encontraron correlaciones estadísticamente significativas para TAV con ambos subgrupos: TAV con CA alto ( $\mathrm{r}=0,61, \mathrm{p}=0,01, \mathrm{n}=15)$, y TAV con CA bajo $(\mathrm{r}=0,74, \mathrm{p}=0,05, \mathrm{n}=9)$.

Similarmente, la grasa abdominal (TAV+TAS) y CA (Tabla 4), mostró correlaciones estadísticamente significativas para CA alto $(\mathrm{r}=0,61)$ y CA bajo $(r=0,74)$. En cambio para TAS no hubo correlación (CA alto $\mathrm{r}=0,33, \mathrm{p}=0,21$ y CA bajo $\mathrm{r}=0,09, \mathrm{p}=0,80)$.

\section{Discusión}

Nuestros resultados indican que las medidas antropométricas habituales tienen un grado de correlación bajo o intermedio con el volumen de grasa abdominal visceral. Esto ocurre tanto en forma global, como discriminando entre aquellos individuos con CA elevada o baja (según criterios

Tabla 3. Análisis estadístico. Correlación de Pearson (r) y valor p, entre TAV y CA, ajustado por cortes definidos para síndrome metabólico ${ }^{14}$ y ajustado por género

\begin{tabular}{|c|c|c|c|c|c|c|c|c|c|}
\hline \multirow[t]{2}{*}{ Variables } & \multicolumn{3}{|c|}{ CA total } & \multicolumn{3}{|c|}{ CA alto } & \multicolumn{3}{|c|}{ CA bajo } \\
\hline & $\begin{array}{c}\text { Total } \\
(n=24)\end{array}$ & $\begin{array}{l}\text { Mujeres } \\
(n=15)\end{array}$ & $\begin{array}{l}\text { Hombres } \\
(n=9)\end{array}$ & $\begin{array}{c}\text { Total } \\
(n=15)\end{array}$ & $\begin{array}{l}\text { Mujeres } \\
(n=12)\end{array}$ & $\begin{array}{l}\text { Hombres } \\
(n=3)\end{array}$ & $\begin{array}{c}\text { Total } \\
(n=9)\end{array}$ & $\begin{array}{c}\text { Mujeres } \\
(n=3)\end{array}$ & $\begin{array}{c}\text { Hombres } \\
(n=6)\end{array}$ \\
\hline TAV & $\begin{array}{l}r=0,56 \\
p<0,01\end{array}$ & $\begin{array}{l}r=0,67 \\
p<0,01\end{array}$ & $\begin{array}{l}r=0,75 \\
p<0,01\end{array}$ & $\begin{array}{l}r=0,61 \\
p<0,01\end{array}$ & $\begin{array}{l}r=0,4 \\
p=0,1\end{array}$ & $\begin{array}{c}r=0,9 \\
p=0,12\end{array}$ & $\begin{array}{l}r=0,74 \\
p<0,05\end{array}$ & $\begin{array}{l}r=0,2 \\
p=0,8\end{array}$ & $\begin{array}{l}r=0,5 \\
p=0,2\end{array}$ \\
\hline
\end{tabular}

TAV: tejido adiposo visceral. CA: circunferencia abdominal. Alto definido como CA $>102 \mathrm{~cm}$ hombres y CA $>88$ mujeres. Bajo definido como $C A \leq 102 \mathrm{~cm}$ hombres y $C A \leq 88 \mathrm{~cm}$ mujeres.

Tabla 4. Análisis estadístico. Correlación de Pearson ( $r$ y valor $p$, entre (TAV+TAS) y CA, ajustado por cortes definidos para síndrome metabólico ${ }^{14}$ y ajustado por género

\begin{tabular}{|ccccccc|}
\hline Variables & Total & $\begin{array}{c}\text { CA alto } \\
\text { Mujeres } \\
(\mathbf{n}=\mathbf{1 5})\end{array}$ & $\begin{array}{c}\text { Hombres } \\
(\mathbf{n}=\mathbf{1 2})\end{array}$ & $\begin{array}{c}\text { Total } \\
(\mathbf{n}=\mathbf{3})\end{array}$ & $\begin{array}{c}\text { Mujeres } \\
\text { Mujo }\end{array}$ & $\begin{array}{c}\text { Hombres } \\
(\mathbf{n}=\mathbf{3})\end{array}$ \\
TAV + TAS & $r=0,62$ & $r=0,72$ & $r=0,94$ & $r=0,7$ & $r=0,4$ & $r=0,6$ \\
& $p<0,01$ & $p<0,01$ & $p=0,2$ & $p<0,05$ & $p=0,7$ & $p=0,16$ \\
\hline
\end{tabular}

(TAV+TAS): grasa abdominal. (CA) circunferencia abdominal. Alto definido como $C A>102 \mathrm{~cm}$ hombres y CA $>88$ mujeres. Bajo definido como $C A \leq 102 \mathrm{~cm}$ hombres y $C A \leq 88 \mathrm{~cm}$ mujeres. 

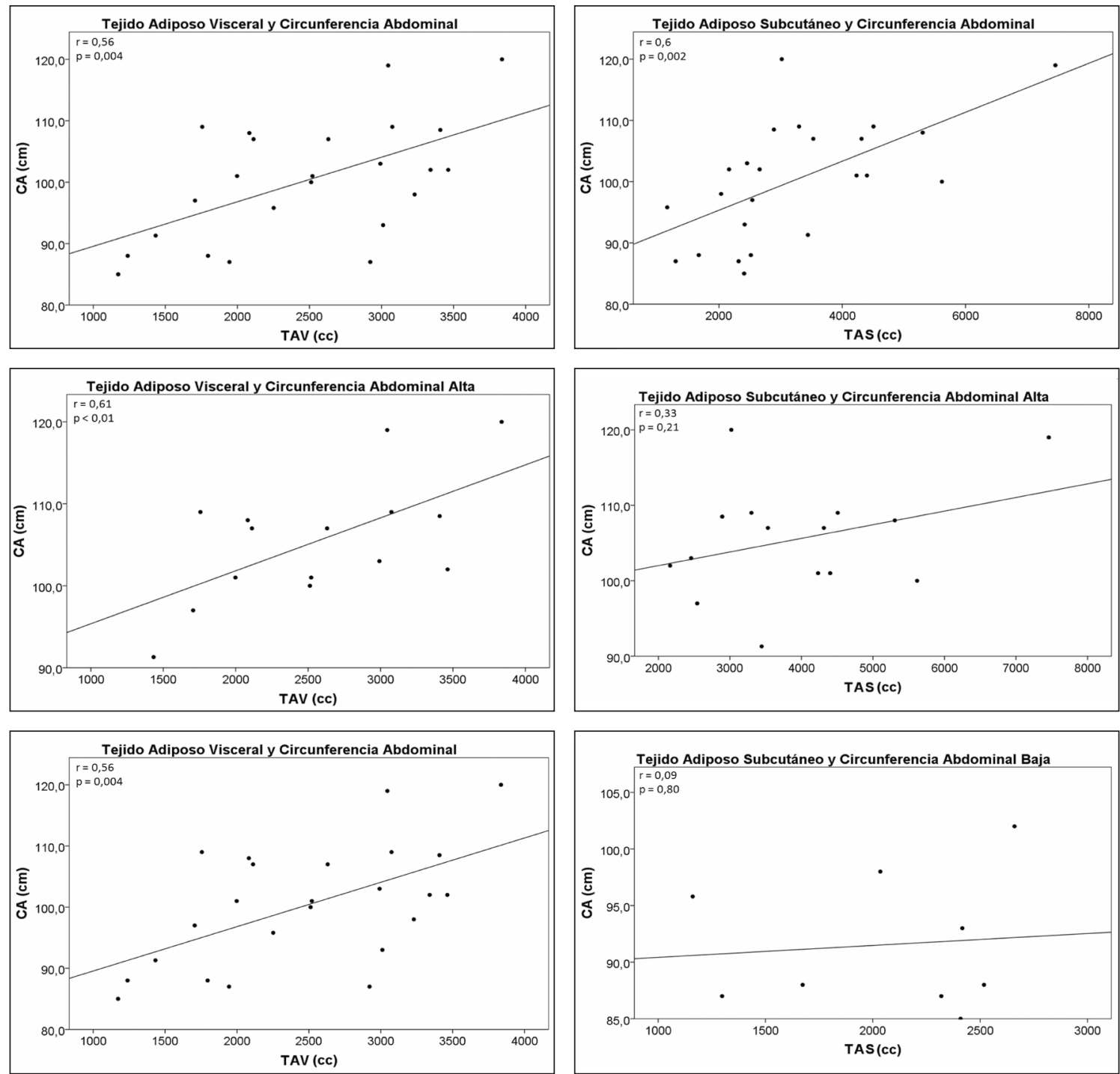

Figura 2. Gráfica de Dispersión Simple. Gráfica entre variables Tejido Adiposo Visceral (TAV), y Tejido Adiposo Subcutáneo (TAS), con la variable antropométrica Circunferencia Abdominal (CA) total, alta y baja, según definición de síndrome metabólico (Hombres $>0 \leq 102 \mathrm{~cm}$, mujeres $>0 \leq 88 \mathrm{~cm}$ respectivamente). a) TAV y CA total. b) TAS y CA total. c) TAV y CA alta. d) TAS y CA alta. e) TAV y CA baja. f) TAS y CA baja.

de síndrome metabólico), e independientemente del sexo. Las medidas de TAS y de TAV+TAS mostraron un mejor grado de correlación con las medidas antropométricas respecto a TAV, con un $\mathrm{r}$ de 0,8 para IMC y CA respectivamente.

Estos hallazgos sugieren que las medidas antropométricas clásicas son mejores indicadores de TAS (IMC) o de TAV+TAS (CA, porcentaje de grasa), y no serían un fiel indicador de la distribución de TAV en pacientes con DM2, quienes tienen más riesgo de tener enfermedades derivadas de la grasa metabólicamente activa, como lo es la esteatosis hepática o enfermedades cardiovasculares ${ }^{41}$. Más aun, el grado de correlación disminuye en sujetos con CA alto, lo que sugiere que este marcador es menos representativo de TAV en los sujetos con mayor volumen abdominal.

Encontrar parámetros antropométricos que 
se correlacionen bien con distribución de TAV es un campo activo de investigación. Hay quienes sostienen por ejemplo que el índice entre la circunferencia de la cintura y la circunferencia de cadera (Índice Cintura/Cadera; ICC) podrían predecir el volumen de $\mathrm{TAV}^{46}$. No obstante, también hay estudios que demuestran exactamente lo contrario $^{16}$. Otros parámetros antropométricos que se han postulado como indicadores de la cantidad de TAV son el índice visceral adiposo ${ }^{47}$ y la cintura hipertrigliceridémica ${ }^{35,48}$.

En este trabajo la medición de grasa por RM se realizó a través de secuencias de excitación espectral de grasa. La ventaja de esta secuencia es que obtiene sólo señal de la grasa, y por lo tanto, permite segmentar fácilmente TAV y TAS. Sin embargo, uno de los problemas de esta secuencia es su sensibilidad a las inhomogeneidades del campo magnético ${ }^{32,49}$. Este artefacto se pudo observar en algunas zonas de TAS, como una pérdida de señal. Para obtener resultados más fidedignos de la cantidad de TAS, se realizó una corrección manual de estos artefactos.

Para solucionar los problemas de inhomogeneidades se han desarrollado recientemente otros métodos. Por ejemplo el método IDEAL ${ }^{\circledR}$ permite obtener imágenes de agua y grasa, corrigiendo además las inhomogeneidades de campo y decaimiento de señal debido a T2 ${ }^{\star 27,32}$. Sin embargo, esta secuencia no está disponible aún para equipos de RM estándares.

Para realizar la medición de grasa, se utilizó el software Image J, que permite desarrollar un protocolo de segmentación de la grasa en forma semi automática. Si bien se logró reducir la duración del procesamiento y los artefactos de las imágenes, el proceso completo para medir TAV y TAS sigue siendo relativamente largo. Es por ello que es necesario desarrollar aplicaciones computacionales que permitan la medición de TAV y TAS en forma más rápida y automática ${ }^{29-32}$.

En conclusión, estimaciones indirectas de TAV a partir de parámetros antropométricos estándares no son aconsejables en pacientes DM2 ya que estas variables presentan un grado de correlación bajo (CA) o no significativo. Dada la relevancia clínica que conlleva la cantidad de TAV, resulta muy útil disponer de una herramienta como la RM para medir adecuadamente la cantidad de grasa intra abdominal y así monitorear mejor los riesgos asociados a las enfermedades metabólicas.

\section{Referencias}

1. Mann GV. The influence of obesity in health. Review Part 1. N Engl J Med 1974; 291: 178-85.

2. Wilson PW, D’Agostino RB, Sullivan L, Parise H, Kannel WB. Overweight and obesity as determinants of cardiovascular risk: the Framingham experience. Arch Intern Med 2002; 162 (16): 1867-72.

3. Manson JE, Colditz GA, Stampfer MJ, Willett WC, Rosner B, Monson RR, et al. A prospective study of obesity and risk of coronary heart disease in women. $\mathrm{N}$ Engl J Med 1990; 322 (13): 882-9.

4. Kenchaiah S, Evans JC, Levy D, Wilson PW, Benjamin EJ, Larson MG et al. Obesity and the risk of heart failure. N Engl J Med 2002; 347 (5): 305-13.

5. Sharma AM, Chetty VT. Obesity, hypertension and insulin resistance. Acta Diabetol 2005; 42 (Suppl 1): S3-S8.

6. Kitabchi AE, Buffington CK. Body fat distribution, hyperandrogenicity, and health risks. Semin Reprod Endocrinol 1994; 12 (1): 6-14.

7. Albu JB, Kovera AJ, Johnson JA. Fat distribution and health in obesity. Ann N Y Acad Sci 2000; 904: 491501.

8. Lapidus L, Bengtsson C, Larsson B, Pennert K, Rybo E, Sjöström L. Distribution of adipose tissue and risk of cardiovascular disease and death: a 12 year follow up of participants in the population study of women in Gothenburg, Sweden. Br Med J (Clin Res Ed) 1984; 289 (6454): 1257-61.

9. Despres JP, Moorjani S, Ferland M, Tremblay A, Lupien PJ, Nadeau A, et al. Adipose tissue distribution and plasma lipoprotein levels in obese women: importance of intra-abdominal fat. Arteriosclerosis 1989; 9 (2): 203-10.

10. Bruunsgaard H, Pedersen BK. Age-related inflammatory cytokines and disease. Immunol Allergy Clin North Am 2003; 23 (1): 15-39.

11 Gastaldelli A, Sironi AM, Ciociaro D, Positano V, Buzzigoli E, Giannessi D et al. Visceral fat and beta cell function in non-diabetic humans. Diabetologia 2006; 48 (10): 2090-6.

12. Expert Panel on the Identification, Evaluation, and Treatment of Overweight in Adults. Clinical guidelines on the identification, evaluation, and treatment of overweight and obesity in adults: executive summary. Am J Clin Nutr 1998; 68 (4): 899-917.

13. WHO. Obesity: preventing and managing the global epidemic: report of a WHO consultation. World Health Organ Tech Rep Ser 2000; 894: 1-253.

14. Executive summary of the third report of the National cholesterol education program (NCEP), expert panel on detection, evaluation, and treatment of high blood 
cholesterol in adults (Adult Treatment Panel III). JAMA 2001; 285 (19): 2486-2497.

15. Ferrannini E, Sironi A, Iozzo P, Gastaldelli A. [Intraabdominal adiposity, abdominal obesity, and cardiometabolic risk]. Eur Heart J Suppl 2008; 10: B4-B10.

16. van der Kooy K, Leenen R, Seidell JC, Deurenberg P, Droop A, Bakker CJ. Waist-hip ratio is a poor predictor of changes in visceral fat. Am J Clin Nutr 1993; 57 (3): 327-33.

17. Roche AF, Sievogel RM, Chumlea WC, Webb P. Grading body fatness from limited anthropometric data. Am J Clin Nutr 1981; 34 (12): 2831-38.

18. Wellens RI, Roche AF, Khamis HJ, Jackson AS, Pollock ML, Siervogel RM. Relationships between the body mass index and body composition. Obes Res 1996; 4 (1): 3544.

19. Ellis KJ. [Human body composition: in vivo methods]. Physiol Rev 2000; 80 (2): 649-80.

20. Seidell JC, Bakker CJ, van der Kooy K. Imaging techniques for measuring adipose-tissue distribution-a comparison between computed tomography and 1.5-T magnetic resonance. Am J Clin Nutr 1990; 51 (6): 953-7.

21. Staten MA, Totty WG, Kohrt WM. Measurement of fat distribution by magnetic resonance imaging. Invest Radiol 1989; 24 (5): 345-9.

22. Lancaster JL, Ghiatas AA, Alyassin A, Kilcoyne RF, Bonora E, De Fronzo RA. Measurement of abdominal fat with T1-weighted MR images. J Magn Reson Imaging 1991; 1 (3): 363-9.

23. Barac-Nieto M, Gupta RK. Use of proton MR spectroscopy and MR imaging to assess obesity. J Magn Reson Imaging 1996; 6 (1): 235-8.

24. Karampinos DC, Yu H, Shimakawa A, Link TM, Majumdar S. T1-Corrected Fat Quantification Using Chemical Shift-Based Water/Fat Separation: Application to Skeletal Muscle. Magn Reson Med 2011; 66 (5): 1312-26.

25. Kaldoudi E, Williams SC, Barker GJ, Tofts PS. A chemical shift selective inversion recovery sequence for fat-suppressed MRI: theory and experimental validation. Magn Reson Imaging 1993; 11 (3): 341-55.

26. Dixon WT. Simple proton spectroscopic imaging. Radiology 1984; 153 (1): 189-94.

27. Reeder SB, McKenzie CA, Pineda AR, Yu H, Shimakawa A, Brau AC et al. Water-fat separation with IDEAL gradient-echo imaging. J Magn Reson Imaging 2007; 25 (3): 644-52.

28. Yu H, McKenzie CA, Shimakawa A, Vu AT, Brau AC, Beatty PJ et al. Multiecho reconstruction for simultaneous water-fat decomposition and $\mathrm{T} 2{ }^{*}$ estimation. J Magn Reson Imaging 2007; 26 (4): 1153-61.

29. Positano V, Gastaldelli A, Sironi AM, Santarelli MF,
Lombardi M, Landini L. An accurate and robust method for unsupervised assessment of abdominal fat by MRI. J Magn Reson Imaging 2004; 20 (4): 684-9.

30. Arif H, Racette SB, Villareal DT, Holloszy JO, Weiss EP. Comparison of methods for assessing abdominal adipose tissue from magnetic resonance images. Obesity (Silver Spring) 2007; 15 (9): 2240-4.

31. Demerath EW, Ritter KJ, Couch WA, Rogers NL, Moreno GM, Choh A et al. Validity of a new automated software program for visceral adipose tissue estimation. Int J Obes (Lond) 2007; 31 (2): 285-91.

32. $\mathrm{Hu} \mathrm{HH}$, Nayak KS, Goran MI. Assessment of abdominal adipose tissue and organ fat content by magnetic resonance imaging. Obes Rev 2011; 12 (5): e504-15.

33. Browning LM, Mugridge O, Dixon AK, Aitken SW, Prentice AM, Jebb SA. Measuring abdominal adipose tissue: comparison of simpler methods with MRI. Obes Facts 2011; 4 (1): 9-15.

34. Bahl M, Qayyum A, Westphalen AC, Noworolski SM, Chu PW, Ferrell L, et al. Liver steatosis: investigation of opposed-phase T1-weighted liver MR signal intensity loss and visceral fat measurement as biomarkers. Radiology 2008; 249 (1): 160-6.

35. Sam S, Haffner S, Davidson MH, D'Agostino RB Sr, Feinstein S, Kondos G, et al. Hypertriglyceridemic waist phenotype predicts increased visceral fat in subjects with type 2 diabetes. Diabetes Care 2009; 32 (10): 1916-20.

36. Bozzetto L, Prinster A, Mancini M, Giacco R, De Natale C, Salvatore M, et al. Liver fat in obesity: role of type 2 diabetes mellitus and adipose tissue distribution. Eur J Clin Invest 2011; 41 (1): 39-44.

37. Miyazaki Y, DeFronzo RA. Visceral fat dominant distribution in male type 2 diabetic patients is closely related to hepatic insulin resistance, irrespective of body type. Cardiovasc Diabetol 2009; 8-44.

38. Kim SK, Park SW, Kim SH, Cha BS, Lee HC, Cho YW. Visceral fat amount is associated with carotid atherosclerosis even in type 2 diabetic men with a normal waist circumference. Int J Obes (Lond) 2009; 33 (1): 131-5.

39. Williamson RM, Price JF, Glancy S, Perry E, Nee LD, Hayes PC et al. Prevalence of and risk factors for hepatic steatosis and nonalcoholic Fatty liver disease in people with type 2 diabetes: the edinburgh type 2 diabetes study. Diabetes Care 2011; 34 (5): 1139-44.

40. Arrese M, Arab JP, Arancibia JP, Candia R, Riquelme A, Barrera F. Liver Disease: A Neglected Complication of Diabetes Mellitus. In: Bagchi D. Nutritional and Therapeutic Interventions for Diabetes and Metabolic Syndrome. Elsevier Inc.: Academic Press; 2012. p. 289-96.

41. Eguchi Y, Mizuta T, Sumida Y, Ishibashi E, Kitajima Y, Isoda $\mathrm{H}$, et al. The pathological role of visceral fat accu- 
mulation in steatosis, inflammation, and progression of nonalcoholic fatty liver disease. J Gastroenterol 2011; 46 Suppl 1: 70-8.

42. van der Poorten D, Milner KL, Hui J, Hodge A, Trenell MI, Kench JG et al. Visceral fat: a key mediator of steatohepatitis in metabolic liver disease. Hepatology 2008; 48 (2): 449-457.

43. American Diabetes Association. Diagnosis and Classification of Diabetes Mellitus. Diabetes Care 2012; 35 (Suppl 1).

44. Durnin JV, Womersley J. Body fat assessed from total body density and its estimation from skinfold thickness: measurements on 481 men and women aged from 16 to 72 years. Br J Nutr 1974; 32 (1): 77-97.

45. Siri WE. Body composition from fluid spaces and density: analysis of methods. 1961. Nutrition 1993; 9 (5): 480-91.
46. Ducluzeau PH, Manchec-Poilblanc P, Roullier V, Cesbron E, Lebigot J, Bertrais S et al. Distribution of abdominal adipose tissue as a predictor of hepatic steatosis assessed by MRI. Clin Radiol 2010; 65 (9): 695-700.

47. Amato MC, Giordano C, Galia M, Criscimanna A, Vitabile S, Midiri M, et al. Visceral Adiposity Index: A reliable indicator of visceral fat function associated with cardiometabolic risk. Diabetes Care 2010; 33 (4): 920-2.

48. Lemieux I, Pascot A, Couillard C, Lamarche B, Tchernof A, Almeras N, et al. Hypertriglyceridemic waist: a marker of the atherogenic metabolic triad (hyperinsulinemia; hyperapolipoprotein B; small, dense LDL) in men? Circulation 2000; 102 (2): 179-84.

49. Peng Q, McColl RW, Ding Y, Wang J, Chia JM, Weatherall PT. Automated method for accurate abdominal fat quantification on water-saturated magnetic resonance images. J Magn Reson Imaging 2007; 26 (3): 738-46. 